\title{
Greedy Shortest Common Superstring Approximation in Compact Space
}

\author{
Alanko, Jarno \\ Springer International Publishing AG \\ 2017-09-06
}

Alanko , J \& Norri , T 2017 , Greedy Shortest Common Superstring Approximation in Compact Space . in G Fici , M Sciortino \& R Venturini (eds), String Processing and Information Retrieval : 24th International Symposium, SPIRE 2017, Palermo, Italy, September 26-29, 2017, Proceedings . Lecture Notes in Computer Science, vol. 10508 , Springer International Publishing AG, Cham , pp. 1-13, International Symposium on String Processing and Information Retrieval , Palermo , Italy , 26/09/2017 . https://doi.org/10.1007/978-3-319-67428-5_1

http://hdl.handle.net/10138/308400

https://doi.org/10.1007/978-3-319-67428-5_1

cc_by

acceptedVersion

Downloaded from Helda, University of Helsinki institutional repository.

This is an electronic reprint of the original article.

This reprint may differ from the original in pagination and typographic detail.

Please cite the original version. 


\title{
Greedy Shortest Common Superstring Approximation in Compact Space
}

\author{
Jarno Alanko and Tuukka Norri \\ Department of Computer Science \\ University of Helsinki \\ Gustaf Hällströmin katu 2b, 00560 Helsinki, Finland \\ jarno.alanko@helsinki.fi, tuukka.norri@helsinki.fi
}

\begin{abstract}
Given a set of strings, the shortest common superstring problem is to find the shortest possible string that contains all the input strings. The problem is NP-hard, but a lot of work has gone into designing approximation algorithms for solving the problem. We present the first time and space efficient implementation of the classic greedy heuristic which merges strings in decreasing order of overlap length. Our implementation works in $O(n \log \sigma)$ time and bits of space, where $n$ is the total length of the input strings in characters, and $\sigma$ is the size of the alphabet. After index construction, a practical implementation of our algorithm uses roughly $5 n \log \sigma$ bits of space and reasonable time for a real dataset that consists of DNA fragments.
\end{abstract}

Keywords: Greedy, Approximation, Compact, Space-efficient, BurrowsWheeler transform, BWT, Shortest Common Superstring, SCS

\section{Introduction}

Given a set of strings, the shortest common superstring is the shortest string which contains each of the input strings as a substsring. The problem is NP-hard [4, but efficient approximation algorithms exist. Perhaps the most practical of the approximation algorithms is the greedy algorithm first analyzed by Tarhio, Ukkonen [14] and Turner [15. The algorithm greedily joins together the pairs of strings with the longest prefix-suffix overlap, until only one string remains. In case there are equally long overlaps, the algorithm can make an arbitrary selection among those. The remaining string is an approximation of the shortest common superstring. The algorithm has been proven to give a superstring with length at most $3 \frac{1}{2}$ times the optimal length [6]. It was originally conjectured by Ukkonen and Tarhio [14] that the greedy algorithm never outputs a superstring that is more than twice as long as the optimal, and the conjecture is still open.

Let $m$ be the number of strings, $n$ be the sum of the lengths of all the strings, and $\sigma$ the size of the alphabet. In 1990 Ukkonen showed how to implement the greedy algorithm in $O(n)$ time and $O(n \log n)$ bits of space using the AhoCorasick automaton 16. Since then, research on the problem has focused on finding algorithms with better provable approximation ratios (see e.g. 9] for a 
summary). Currently, algorithm with the best proven approximation ratio in peer reviewed literature is the one by Mucha with an approximation ratio of $2 \frac{11}{23}$ [9], and there is a preprint claiming an algorithm with a ratio of $2 \frac{11}{30}$ [11. However, we are not aware of any published algorithm that solves the problem in better than $O(n \log n)$ bits of space. Improving the factor $\log n$ to $\log \sigma$ is important in practice. Many of the largest data sets available come from DNA strings which have an alphabet of size only 4 , while $n$ can be over $10^{9}$.

We present an algorithm that implements the greedy heuristic in $O(n \log \sigma)$ time and bits of space. It is based on the FM-index enhanced with a succinct representation of the topology of the suffix tree. The core of the algorithm is the iteration of prefix-suffix overlaps of input strings in decreasing order of length using a technique described in [8] and [13, combined with Ukkonen's bookkeeping [16] to keep track of the paths formed in the overlap graph of the input strings. The main technical novelty of this work is the implementation of Ukkonen's bookkeeping in $O(n \log \sigma)$ space. We also have a working implementation of the algorithm based on the SDSL-library [5]. For practical reasons the implementation differs slightly from the algorithm presented in this paper, but the time and space usage should be similar.

\section{Preliminaries}

Let there be $m$ strings $s_{1}, \ldots, s_{m}$ drawn from the alphabet $\Sigma$ of size $\sigma$ such that the sum of the lengths of the strings is $\sum_{i=1}^{m}\left|s_{i}\right|=n$. We build a single string by concatenating the $m$ strings, placing a separator character $\$ \notin \Sigma$ between each string. We define that the separator is lexicographically smaller than all characters in $\Sigma$. This gives us the string $S=s_{1} \$ s_{2} \$ \cdots s_{m} \$$ of length $n+m$. Observe that the set of suffixes that are prefixed by some substring $\alpha$ of $S$ are adjacent in the lexicographic ordering of the suffixes. We call this interval in the sorted list of suffixes the lexicographic range of string $\alpha$. All occurrences of a substring $\alpha$ can be uniquely represented as a triple $\left(a_{\alpha}, b_{\alpha}, d_{\alpha}\right)$, where $\left[a_{\alpha}, b_{\alpha}\right]$ is the lexicographic range of $\alpha$, and $d_{\alpha}$ is the length of $\alpha$. A string $\alpha$ is right maximal in $S$ if and only if there exist two or more distinct characters $y, z \in \Sigma \cup\{\$\}$ such that the strings $\alpha y$ and $\alpha z$ are substrings of $S$. Our algorithm needs support for two operations on substrings: left extensions and suffix links. A left extension of string $\alpha$ with character $x$ is the map $\left(a_{\alpha}, b_{\alpha}, d_{\alpha}\right) \mapsto\left(a_{x \alpha}, b_{x \alpha}, d_{x \alpha}\right)$. A suffix link for the right-maximal string $x \alpha$ is the map $\left(a_{x \alpha}, b_{x \alpha}, d_{x \alpha}\right) \mapsto\left(a_{\alpha}, b_{\alpha}, d_{\alpha}\right)$.

\section{Overview of the Algorithm}

We use Ukkonen's 1990 algorithm [16] as a basis for our algorithm. Conceptually, we have a complete directed graph where vertices are the input strings, and the weight of the edge from string $s_{i}$ to string $s_{j}$ is the length of the longest suffix of $s_{i}$ which is also a prefix of $s_{j}$. If there is no such overlap, the weight of the edge is zero. The algorithm finds a Hamiltonian path over the graph, and merges the strings in the order given by the path to form the superstring. We define the 
merge of strings $s_{i}=\alpha \beta$ and $s_{j}=\beta \gamma$, where $\beta$ is the longest prefix-suffix overlap of $s_{i}$ and $s_{j}$, as the string $\alpha \beta \gamma$. It is known that the string formed by merging the strings in the order given by the maximum weight Hamiltonian path gives a superstring of optimal length [14. The greedy algorithm tries to heuristically find a Hamiltonian path with a large total length.

Starting from a graph $G$ where the vertices are the input strings and there are no edges, the algorithm iterates all prefix-suffix overlaps of pairs of strings in decreasing order of length. For each pair $\left(s_{i}, s_{j}\right)$ we add an edge from $s_{i}$ to $s_{j}$ iff the in-degree of $s_{j}$ is zero, the out-degree of $s_{i}$ is zero, and adding the edge would not create a cycle in $G$. We also consider overlaps of length zero, so every possible edge is considered and it is easy to see that in the end the added edges form a Hamiltonian path over $G$.

\section{Algorithm}

Observe that if an input string is a proper substring of another input string, then any valid superstring that contains the longer string also contains the shorter string, so we can always discard the shorter string. Similarly if there are strings that occur multiple times, it suffices to keep only one copy of each. This preprocessing can be easily done in $O(n \log \sigma)$ time and space for example by backward searching all the input strings using the FM-index.

After the preprocessing, we sort the input strings into lexicographic order, concatenate them placing dollar symbols in between the strings, and build an index that supports suffix links and left extensions. The sorting can be done with merge sort such that string comparisons are done $O(\log (n))$ bits at a time using machine word level parallelism, as allowed by the RAM model. This works in $O(n \log \sigma)$ time and space if the sorting is implemented so that it does not move the strings around, but instead manipulates only pointers to the strings.

For notational convenience, from here on $s_{i}$ refers to the string with lexicographic rank $i$ among the input strings.

We iterate in decreasing order of length all the suffixes of the input strings $s_{i}$ that occur at least twice in $S$ and for each check whether the suffix is also a prefix of some other string $s_{j}$, and if so, we add an edge from $s_{i}$ to $s_{j}$ if possible. To enumerate the prefix-suffix overlaps, we use the key ideas from the algorithm for reporting all prefix-suffix overlaps to build an overlap graph described in [8] and [13, adapted to get the overlaps in decreasing order of length.

We maintain an iterator for each of the input strings. An iterator for the string $s_{i}$ is a quadruple $(i, \ell, r, d)$, where $[\ell, r]$ is the lexicographic range of the current suffix $\alpha$ of $s_{i}$ and $d$ is the length of $\alpha$, i.e. the depth of the iterator. Suffixes of the input strings which are not right maximal in the concatenation $S=s_{1} \$ \ldots s_{m} \$$ can never be a prefix of any of the input strings. The reason is that if $\alpha$ is not right-maximal, then $\alpha$ is always followed by the separator $\$$. This means that if $\alpha$ is also a prefix of some other string $s_{j}$, then $s_{j}=\alpha$, because the only prefix of $s_{j}$ that is followed by a $\$$ is the whole string $s_{j}$. But then $s_{j}$ is a substring of $s_{i}$, which can not happen because all such strings were removed 
in the preprocessing stage. Thus, we can safely disregard any suffix $\alpha$ of $s_{i}$ that is not right maximal in $S$. Furthermore, if a suffix $\alpha$ of $s_{i}$ is not right maximal, then none of the suffixes $\beta \alpha$ are right-maximal either, so we can disregard those, too.

We initialize the iterator for each string $s_{i}$ by backward searching $s_{i}$ using the FM-index for as long as the current suffix of $s_{i}$ is right-maximal. Next we sort these quadruples in the decreasing order of depth into an array iterators. When this is done, we start iterating from the iterator with the largest depth, i.e. the first element of iterators. Suppose the current iterator corresponds to string $i$, and the current suffix of string $s_{i}$ is $\alpha$. At each step of the iteration we check whether $\alpha$ is also a prefix of some string by executing a left extension with the separator character $\$$. If the lexicographic range $\left[\ell^{\prime}, r^{\prime}\right]$ of $\$ \alpha$ is nonempty, we know that the suffixes of $S$ in the range $\left[\ell^{\prime}, r^{\prime}\right]$ start with a dollar and are followed by a string that has $\alpha$ as a prefix. We conclude that the input string with lexicographic rank $i$ among the input strings has a suffix of length $d$ that matches a prefix of the strings with lexicographic ranks $\ell^{\prime}, \ldots, r^{\prime}$ among the input strings. This is true because the lexicographic order of the suffixes of $S$ that start with dollars coincides with the lexicographic ranks of the strings following the dollars in the concatenation, because the strings are concatenated in lexicographic order.

Thus, according to the greedy heuristic, we should try to merge $s_{i}$ with a string from the set $s_{\ell^{\prime}}, \ldots, s_{r^{\prime}}$, which corresponds to adding an edge from $s_{i}$ to some string from $s_{\ell^{\prime}}, \ldots, s_{r^{\prime}}$ in the graph $G$. We describe how we maintain the graph $G$ in a moment. After updating the graph, we update the current iterator by decreasing $d$ by one and taking a suffix link of the lexicographic range $[\ell, r]$. The iterator with the next largest $d$ can be found in constant time because the array iterators is initially sorted in descending order of depth. We can maintain a pointer to the iterator with the largest $d$. If at some step iterators $[k]$ has the largest depth, then in the next step either iterators $[k+1]$ or iterators[1] has the largest depth. The pseudocode for the main iteration loop is shown in Algorithm 1.

Now we describe how we maintain the graph $G$. The range $\left[\ell^{\prime}, r^{\prime}\right]$ now represents the lexicographical ranks of the input strings that are prefixed by $\alpha$ among all input strings. Each string $s_{j}$ in this range is a candidate to merge to string $s_{i}$, but some bookkeeping is needed to keep track of available strings. We use essentially the same method as Tarhio and Ukkonen [14. We have bit vectors leftavailable $[1 . . m]$ and rightavailable $[1 . . m]$ such that leftavailable $[k]=$ 1 if and only if string $s_{k}$ is available to use as the left side of a merge, and rightavailable $[k]=1$ if and only if string $s_{k}$ is available as the right side of a merge. Equivalently, leftavailable $[k]=1$ iff the out-degree of $s_{k}$ is zero and rightavailable $[k]=1$ if the in-degree of $s_{k}$ is zero. Also, to prevent the formation of a cycle, we need arrays leftend[1..m], where leftend $[k]$ gives the leftmost string of the chain of merged strings to the left of $s_{k}$, and rightend[1..m], where rightend $[k]$ gives the rightmost string of the chain of merged strings to 


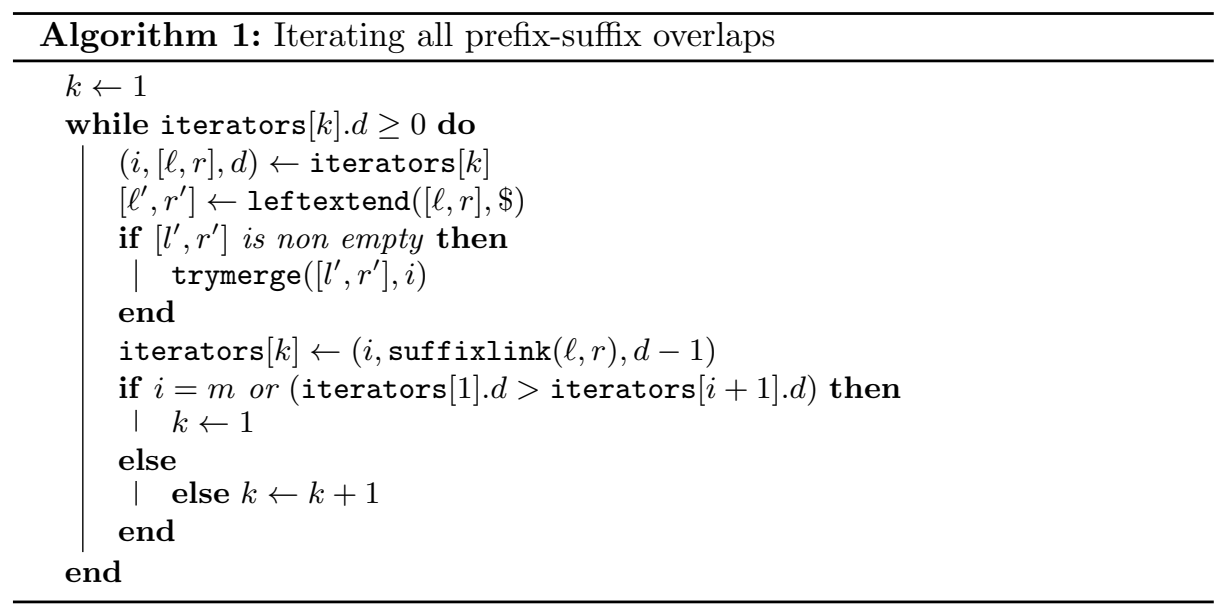

the right of $s_{k}$. We initialize leftavailable $[k]=$ rightavailable $[k]=1$ and leftend $[k]=\operatorname{rightend}[k]=k$ for all $k=1, \ldots, m$.

When we get the interval $\left[\ell^{\prime}, r^{\prime}\right]$ such that leftavailable $[j]=1$, we try to find an index $j \in\left[\ell_{\$ \alpha}, r_{\$ \alpha}\right]$ such that rightavailable $[i]=1$ and leftend $[j] \neq$ $i$. Luckily we only need to examine at most two indices $j$ and $j^{\prime}$ such that rightavailable $[j]=1$ and rightavailable $\left[j^{\prime}\right]=1$ because if leftend $[j]=i$, then leftend $\left[j^{\prime}\right] \neq i$, and vice versa. This procedure is named trymerge $\left(\left[l^{\prime}, r^{\prime}\right], i\right)$ in Algorithm 1.

The problem is now to find up to two ones in the bit vector rightavailable in the interval of indices $\left[\ell_{\$ \alpha}, r_{\$ \alpha}\right]$. To do this efficiently, we maintain for each index $k$ in rightavailable the index of the first one in rightavailable $[k+$ 1..m], denoted with next_one $(k)$. If there are two ones in the interval $\left[\ell_{\$ \alpha}, r_{\$ \alpha}\right]$, then they can be found at next_one $\left(\ell_{{ }_{\alpha}}-1\right)$ and next_one(next_one $\left.\left(\ell_{{ }_{\alpha}}-1\right)\right)$. The question now becomes, how do we maintain this information efficiently? In general, this is the problem of indexing a bit vector for dynamic successor queries, for which there does not exist a constant time solution using $O(n \log \sigma)$ space in the literature. However, in our case the vector rightavailable starts out filled with ones, and once a one is changed to a zero, it will not change back for the duration of the algorithm, which allows us to have a simpler and more efficient data structure.

Initially, next_one $(k)=k+1$ for all $k<m$. The last index does not have a successor, but it can easily be handled as a special case. For clarity and brevity we describe the rest of the process as if the special case did not exist. When we update $\operatorname{rightavailable}(k):=0$, then we need to also update next_one $\left[k^{\prime}\right]:=$ next_one $(k)$ for all $k^{\prime}<k$ such that rightavailable $\left[k^{\prime}+1 . . k\right]$ contains only zeros. To do this efficiently, we store the value of next_one only once for each sequence of consecutive zeros in rightavailable, which allows us to update the whole range at once. To keep track of the sequences of consecutive zeros, we can use a union-find data structure. A union-find data structure 
maintains a partitioning of a set of elements into disjoint groups. It supports the operations find $(x)$, which returns the representative of the group containing $x$, and union $(x, y)$, which takes two representatives and merges the groups containing them.

We initialize the union-find structure such that there is an element for every index in rightavailable, and we also initialize an array next $[1 . . m]$ such that next $[k]:=k+1$ for all $k=1, \ldots m$. When a value at index $k$ is changed to a zero, we compute $q:=\operatorname{next}[\operatorname{find}(k)]$. Then we will do union $(\operatorname{find}(k), \operatorname{find}(k-1))$ and if rightavailable $[k+1]=0$, we will do $\operatorname{union}(\operatorname{find}(k), \operatorname{find}(k+1))$. Finally, we update next $[\mathrm{find}(k)]=q$. We can answer queries for next_one $(k)$ with next $[\mathrm{f} \operatorname{ind}(k)]$.

Whenever we find a pair of indices $i$ and $j$ such that leftavailable $[i]=1$, rightavailable $[j]=1$ and leftend $[j] \neq i$, we add an edge from $s_{i}$ to $s_{j}$ by recording string $j$ as the successor of string $i$ using arrays successor $[1 . . m]$ and overlaplength $[1 . . m]$. We set successor $[j]=i$ and overlaplength $[j]=d_{i}$, where $d_{i}$ is the length of the overlap of $s_{i}$ and $s_{j}$, and do the updates:

$$
\begin{aligned}
\text { leftavailable }[i] & :=0 \\
\text { rightavailable }[j] & :=0 \\
\text { leftend }[\text { rightend }[j]] & :=\text { leftend }[i] \\
\text { rightend }[\text { leftend }[i]] & :=\operatorname{rightend}[j]
\end{aligned}
$$

Note that the arrays leftend and rightend are only up to date for the end points of the paths, but this is fine for the algorithm. Finally we update the next array with the union-find structure using the process described earlier. We stop iterating when we have done $m-1$ merges. At the end, we have a Hamiltonian path over $G$, and we form a superstring by merging the strings in the order specified by the path.

\section{Time and Space Analysis}

The following space analysis is in terms of number of bits used. We assume that the strings are binary encoded such that each character takes $\left\lceil\log _{2} \sigma\right\rceil$ bits. A crucial observation is that we can afford to store a constant number of $O(\log n)$ bit machine words for each distinct input string.

Lemma 1. Let there be $m$ distinct non-empty strings with combined length $n$ from an alphabet of size $\sigma>1$. Then $m \log n \in O(n \log \sigma)$.

Proof. Suppose $m \leq \sqrt{n}$. Then the Lemma is clearly true, because:

$$
m \log n \leq \sqrt{n} \log n \in O(n \log \sigma)
$$

We now consider the remaining case $m \geq \sqrt{n}$, or equivalently $\log n \leq 2 \log m$. This means $m \log n \leq 2 m \log m$, so it suffices to show $m \log m \in O(n \log \sigma)$. 
First, note that at least half of the strings have length at least $\log (m)-1$ bits. This is trivially true when $\log (m)-1 \leq 1$. When $\log (m)-1 \geq 2$, the number of distinct binary strings of length at most $\log (m)-2$ bits is

$$
\sum_{i=1}^{\lfloor\log (m)-2\rfloor} 2^{i} \leq 2^{\log (m)-1}=\frac{1}{2} m
$$

Therefore indeed at least half of the strings have length of at least $\log m-1$ bits. The total length of the strings is then at least $\frac{1}{2} m(\log m-1)$ bits. Since the binary representation of all strings combined takes $n\left\lceil\log _{2} \sigma\right\rceil$ bits, we have $n\left\lceil\log _{2} \sigma\right\rceil \geq$ $\frac{1}{2} m(\log m-1)$, which implies $m \log m \leq 2 n\left\lceil\log _{2} \sigma\right\rceil+1 \in O(n \log \sigma)$.

Next, we describe how to implement the suffix links and left extensions. We will need to build the following data structures for the concatenation of all input strings separated by a separator character:

- The Burrows-Wheeler transform, represented as a wavelet tree with support for rank and select queries.

- The $C$-array, which has length equal to the number of characters in the concatenation, such that $C[i]$ is the number of occurrences of characters with lexicographic rank strictly less than $i$.

- The balanced parenthesis representation of the suffix tree topology with support for queries for leftmost leaf, rightmost leaf and lowest common ancestor.

Note that in the concatenation of the strings, the alphabet size is increased by one because of the added separator character, and the total length of the data in characters is increased by $m$. However this does not affect the asymptotic size of the data, because

$$
(n+m) \log (\sigma+1) \leq 2 n(\log \sigma+1) \in \Theta(n \log \sigma)
$$

The three data structures can be built and represented in $O(n \log \sigma)$ time and space [1. Using these data structures we can implement the left extension for lexicographic interval $[\ell, r]$ with the character $c$ by:

$$
([\ell, r], c) \mapsto\left[C[c]+\operatorname{rank}_{B W T}(\ell, c), C[c]+\operatorname{rank}_{B W T}(r, c)\right]
$$

We can implement the suffix link for the right maximal string $c \alpha$ with the lexicographic interval $[\ell, r]$ by first computing

$$
v=1 \mathrm{ca}\left(\operatorname{select}_{B W T}(c, \ell-C[c]), \operatorname{select}_{B W T}(c, r-C[c])\right)
$$

and then

$$
[\ell, r] \mapsto[\text { leftmostleaf }(v), \text { rightmostleaf }(v)]
$$

This suffix link operation works as required for right-maximal strings by removing the first character of the string, but the behaviour on non-right-maximal strings is slightly different. The lexicographic range of a non-right-maximal string is the same as the lexicographic range of the shortest right-maximal string that 
has it as a prefix. In other words, for a non-right-maximal string $c \alpha$, the operation maps the interval $\left[\ell_{c \alpha}, r_{c \alpha}\right]$ to the lexicographic interval of the string $\alpha \beta$, where $\beta$ is the shortest right-extension that makes $c \alpha \beta$ right-maximal. This behaviour allows us to check the right-maximality of a substring $c \alpha$ given the lexicographic ranges $\left[\ell_{\alpha}, r_{\alpha}\right]$ and $\left[\ell_{c \alpha}, r_{c \alpha}\right]$ in the iterator initialization phase of the algorithm as follows:

Lemma 2. The substring ca is right maximal if and only if the suffix link of $\left[\ell_{c \alpha}, r_{c \alpha}\right]$ is $\left[\ell_{\alpha}, r_{\alpha}\right]$.

Proof. As discussed above, the suffix link of $\left[\ell_{c \alpha}, r_{c \alpha}\right]$ maps to the lexicographic interval of the string $\alpha \beta$ where $\beta$ is the shortest right-extension that makes $c \alpha \beta$ right-maximal. Suppose first that $c \alpha$ is right-maximal. Then $\left[\ell_{\alpha \beta}, r_{\alpha \beta}\right]=\left[\ell_{\alpha}, r_{\alpha}\right]$, because $\beta$ is an empty string. Suppose on the contrary that $c \alpha$ is not rightmaximal. Then $\left[\ell_{\alpha \beta}, r_{\alpha \beta}\right] \neq\left[\ell_{\alpha}, r_{\alpha}\right]$, because $\alpha \beta$ and $\alpha$ are distinct right-maximal strings.

Now we are ready to prove the time and space complexity of the whole algorithm.

Theorem 3. The algorithm in Section 4 can be implemented in $O(n \log \sigma)$ time and $O(n \log \sigma)$ bits of space.

Proof. The preprocessing to remove contained and duplicate strings can be done in $O(n \log \sigma)$ time and space for example by building an FM-index, and backward searching all input strings.

The algorithm executes $O(n)$ left extensions and suffix links. The time to take a suffix link is dominated by the time do the select query, which is $O(\log \sigma)$, and the time to do a left extension is dominated by the time to do a rank-query which is also $O(\log \sigma)$. For each left extension the algorithm does, it has to access and modify the union-find structure. Normally this would take amortized time related to the inverse function of the Ackermann function [2], but in our case the amortized complexity of the union-find operations can be made linear using the construction of Gabow and Tarjan [3, because we know that only elements corresponding to consecutive positions in the array rightavailable will be joined together. Therefore, the time to do all left extensions, suffix links and updates to the union-find data structure is $O(n \log \sigma)$.

Let us now turn to consider the space complexity. For each input string, we have the quadruple $(i, \ell, r, d)$ of positive integers with value at most $n$. The quadruples take space $3 m \log m+m \log n$. The union-find structure of Gabow and Tarjan can be implemented in $O(m \log m)$ bits of space [3]. The bit vectors leftavailable and rightavailable take exactly $2 m$ bits, and the arrays successor, leftend, rightend and next take $m \log m$ bits each. The array overlaplength takes $m \log n$ bits of space. Summing up, in addition to the data structures for the left extensions and contractions, we have only $O(m \log n)$ bits of space, which is $O(n \log \sigma)$ by Lemma 1 . 


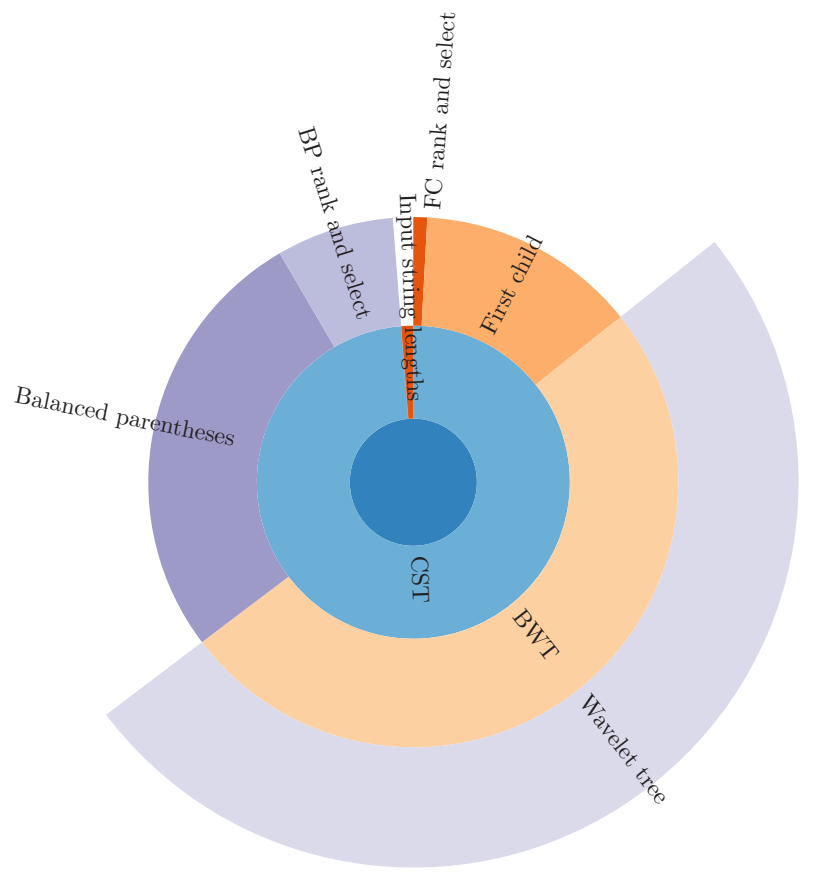

Fig. 1: Memory breakdown of the data structures used by our implementation. The plot was generated using the SDSL library. Each sector angle represents the portion of the memory taken by the data structure of the total memory of the inner data structure; areas have no special meaning. Abbreviations: CST = compressed suffix tree, BWT $=$ Burrows-Wheeler Transform, BP = balanced parenthesis, $\mathrm{FC}=$ first child.

\section{Implementation}

The algorithm was implemented with the SDSL library [5]. A compressed suffix tree that represents nodes as lexicographic intervals [10] was used to implement the suffix links and left extensions. Only the required parts of the suffix tree were built: the FM-index, balanced parentheses support and a bit vector that indicates the leftmost child node of each node. These data structures differ slightly from the description in Section 5, because they were chosen for convenience as they were readily available in the SDSL library, and they should give very similar performance compared to those used in the aforementioned Section. In particular, the leftmost child vector was needed to support suffix links, but we could manage without it by using the operations on the balanced parenthesis support described in Section 5. Our implementation is available at the URL https://github.com/tsnorri/compact-superstring 


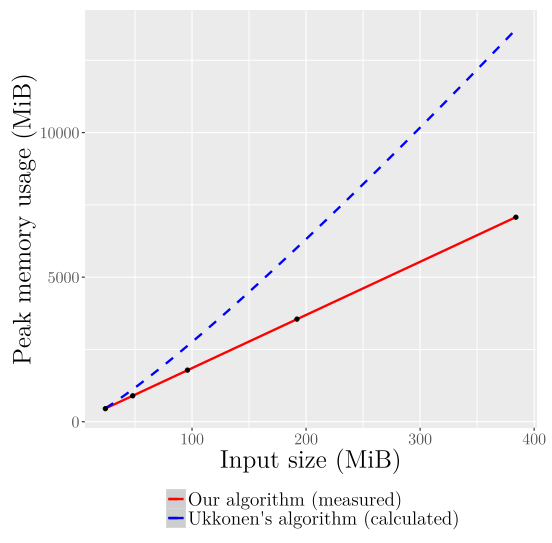

(a) Peak memory

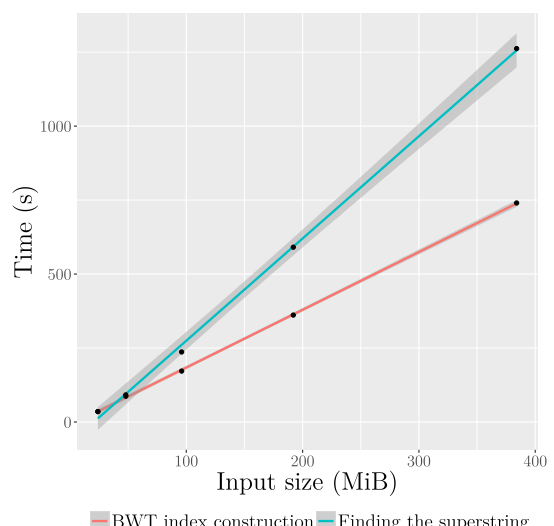

(b) Time consumption

Fig. 2: (a) The peak memory usage of our algorithm plotted against a conservative estimate of $4 n \log n$ bits of space needed by Ukkonen's Aho-Corasick based method. (b) the time usage of our algorithm for the two phases of the algorithm. The data points have been fitted with a least-squares linear model, and the grey band shows the $95 \%$ confidence interval (large enough to be visible only for the second phase). The time and memory usage were measured using the /usr/bin/time command and the RSS value.

The input strings are first sorted with quicksort. This introduces a $\log n$ factor to the time complexity, but it is fast in practice. The implementation then runs in two passes. First, exact duplicate strings are removed and the stripped compact suffix tree is built from the remaining strings. The main algorithm is implemented in the second part. The previously built stripped suffix tree is loaded into memory and is used to find the longest right-maximal suffix of each string and to iterate the prefix-suffix overlaps. Simultaneously, strings that are substrings of other strings are marked for exclusion from building the superstring.

For testing, we took a metagenomic DNA sample from a human gut microbial gene catalogue project 12 , and sampled DNA fragments to create five datasets with $2^{26+i}$ characters respectively for $i=0, \ldots, 4$. The alphabet of the sample was $\{A, C, G, T, N\}$. Time and space usage for all generated datasets for both the index construction phase and the superstring construction phase are plotted in Figure 2. The machine used run Ubuntu Linux version 16.04.2 and has 1.5 TB of RAM and four Intel Xeon CPU E7-4830 v3 processors (48 total cores, $2.10 \mathrm{GHz}$ each). A breakdown of the memory needed for the largest dataset for the different structures comprising the index is shown in Figure 1.

While we don't have an implementation of Ukkonen's greedy superstring algorithm, have a conservative estimate for how much space it would take. The algorithm needs at least the goto- and failure links for the Aho-Corasick automaton, which take at least $2 n \log n$ bits total. The main algorithm uses linked 


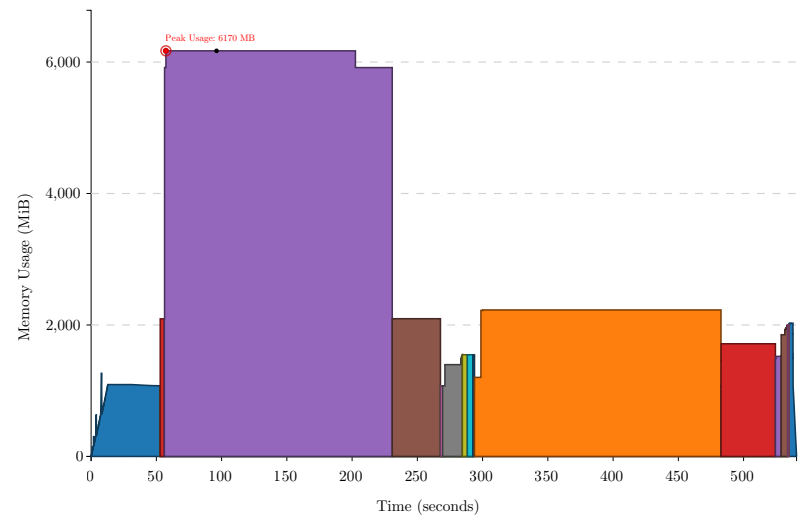

(a) Index construction

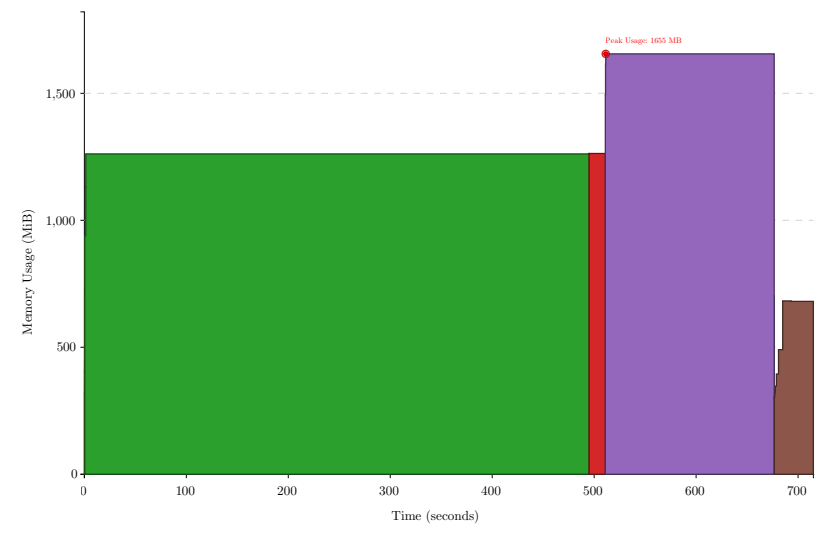

(b) Superstring construction

Fig. 3: Subfigures (a) and (b) show the memory usage as a function of time for index construction and superstring construction, respectively. The peak in Figure (a) occurs during suffix array construction, and the peak in Figure (b) occurs during the iteration of prefix-suffix overlaps.

lists named $L$ and $P$, which take at least $2 n \log n$ bits total. Therefore the space usage is at the very least $4 n \log n$. This estimate is plotted in Figure 2 .

Figure 3 shows the space usage of our algorithm in the largest test dataset as a function of time reported by the SDSL library. The peak memory usage of the whole algorithm occurs during index construction, and more specifically during the construction of a compressed suffix array. The SDSL library used this data structure to build the BWT and the balanced parenthesis representation, which makes the space usage unnecessarily high. This could be improved by using more efficient algorithms to build the BWT and the balanced parenthesis 
representation of the suffix tree topology [1]. These could be plugged in to bring down the index construction memory. The peak memory of the part of the algorithm which constructs the superstring is only approximately 5 times the size of the input in bits.

\section{Discussion}

We have shown a practical way to implement the greedy shortest common superstring algorithm in $O(n \log \sigma)$ time and bits of space. After index construction, the algorithm consists of two relatively independent parts: reporting prefix-suffix overlaps in decreasing order of lengths, and maintaining the overlap graph to prevent merging a string to one direction more than once and the formation of cycles. The part which reports the overlaps could also be done in other ways, such as using compressed suffix trees or arrays, or a succinct representation of the Aho-Corasick automaton. The only difficult part is to avoid having to hold $\Omega(n)$ integers in memory at any given time. We believe it is possible to engineer algorithms using these data structures to achieve $O(n \log \sigma)$ space as well.

Regrettably, we could not find any linear time implementations of Ukkonen's greedy shortest common superstring algorithm for comparison. There is an interesting implementation by Liu and Sýkora [7, but it is too slow for our purposes because it involves computing all pairwise overlap lengths of the input strings to make better choices in resolving ties in the greedy choices. While their experiments indicate that this improves the quality of the approximation, the time complexity is quadratic in the number of input strings. Zaritsky and Sipper [17] also have an implementation of the greedy algorithm, but it's not publicly available, and the focus of the paper is on approximation quality, not performance. As future work, it would be interesting to make a careful implementation of Ukkonen's greedy algorithm, and compare it to ours experimentally.

\section{Acknowledgements}

We would like to thank anonymous reviewers for improving the presentation of the paper.

\section{References}

1. Belazzougui, D.: Linear time construction of compressed text indices in compact space. In: Proceedings of the 46th Annual ACM Symposium on Theory of Computing. pp. 148-193. ACM (2014)

2. Cormen, T.H., Leiserson, C.E., Rivest, R.L., Stein, C.: Introduction to algorithms, vol. 6. MIT press Cambridge (2001)

3. Gabow, H.N., Tarjan, R.E.: A linear-time algorithm for a special case of disjoint set union. Journal of computer and system sciences 30(2), 209-221 (1985)

4. Gallant, J., Maier, D., Astorer, J.: On finding minimal length superstrings. Journal of Computer and System Sciences 20(1), 50-58 (1980) 
5. Gog, S., Beller, T., Moffat, A., Petri, M.: From theory to practice: Plug and play with succinct data structures. In: International Symposium on Experimental Algorithms. pp. 326-337. Springer (2014)

6. Kaplan, H., Shafrir, N.: The greedy algorithm for shortest superstrings. Information Processing Letters 93(1), 13-17 (2005)

7. Liu, X., Sykora, O.: Sequential and parallel algorithms for the shortest common superstring problem. In: Proceedings of the International Workshop on Parallel Numerics. pp. 97-107 (2005)

8. Mäkinen, V., Belazzougui, D., Cunial, F., Tomescu, A.I.: Genome-Scale Algorithm Design. Cambridge University Press (2015)

9. Mucha, M.: Lyndon words and short superstrings. In: Proceedings of the TwentyFourth Annual ACM-SIAM Symposium on Discrete Algorithms. pp. 958-972. Society for Industrial and Applied Mathematics (2013)

10. Ohlebusch, E., Fischer, J., Gog, S.: Cst++. In: International Symposium on String Processing and Information Retrieval. pp. 322-333. Springer (2010)

11. Paluch, K.: Better approximation algorithms for maximum asymmetric traveling salesman and shortest superstring. arXiv preprint arXiv:1401.3670 (2014)

12. Qin, J., Li, R., Raes, J., Arumugam, M., Burgdorf, K.S., Manichanh, C., Nielsen, T., Pons, N., Levenez, F., Yamada, T., et al.: A human gut microbial gene catalogue established by metagenomic sequencing. Nature 464(7285), 59-65 (2010)

13. Simpson, J.T., Durbin, R.: Efficient construction of an assembly string graph using the fm-index. Bioinformatics 26(12), i367-i373 (2010)

14. Tarhio, J., Ukkonen, E.: A greedy approximation algorithm for constructing shortest common superstrings. Theoretical computer science 57(1), 131-145 (1988)

15. Turner, J.S.: Approximation algorithms for the shortest common superstring problem. Information and computation 83(1), 1-20 (1989)

16. Ukkonen, E.: A linear-time algorithm for finding approximate shortest common superstrings. Algorithmica 5(1-4), 313-323 (1990)

17. Zaritsky, A., Sipper, M.: The preservation of favored building blocks in the struggle for fitness: The puzzle algorithm. IEEE Transactions on Evolutionary Computation $8(5), 443-455$ (2004) 